\title{
A rare consortium of functional neurological disorder and Fahr's disease - a herald of extensive calcification
}

\author{
Alka Chauhan', Sunny Garg ${ }^{2}$ \\ ${ }^{1}$ Junior Resident, ${ }^{2}$ Senior Resident, Department of Psychiatry, Bhagat Phool Singh Medical College For Women, \\ Sonipat, Haryana, India
}

\section{A B S T R A C T}

Fahr's disease is also known as familial idiopathic basal ganglia calcification affecting the cerebral microvessels and presenting with numerous neuropsychiatric manifestations. Functional neurological disorder (FND), a pure neuropsychiatric phenomenon occurs rarely in Fahr's disease. Herein, we have presented a case of 17 year old female presenting neuropsychiatric symptoms like pseudoseizure, left sensorineural deficit and mild headache for last 4 months. Mental status examination revealed impaired attention and concentration. Laboratory examinations were within normal limits except CT scan and MRI Brain which revealed bilateral and symmetrical basal ganglia calcification. This is a rare case of FND associated with Fahr's Disease, never reported in the literature. This case highlights the need for evaluating the biological factors in causation of FND where psychological factors were not assessed.

Key words: Basal ganglia calcification; Bilateral; Fahr's disease; FND; Neuropsychiatric symptoms

\section{Access this article online}

Website:

http://nepjol.info/index.php/AJMS DOI: 10.3126/ajms.v11i6.30632

E-ISSN: 2091-0576

P-ISSN: 2467-9100

Copyright (c) 2020 Asian Journal of Medical Sciences

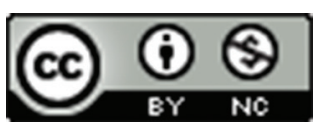

This work is licensed under a Creative Commons Attribution-NonCommercial 4.0 International License.

\section{INTRODUCTION}

Fahr's disease (FD) is a rare inherited neurological disorder characterised by neuropsychiatric, movement and cognitive disorders associated with bilateral symmetrical basal ganglia calcification which is idiopathic in nature. There is strong evidence for autosomal dominant mode of inheritance or inheritance may be autosomal recessive or sporadic. The prevalence of FD is $1 / 1,000,000$. Fahr's disease typically affects the individual in their $3^{\text {rd }}$ or $4^{\text {th }}$ decade of life and rarely present in adolescents. ${ }^{1}$ FD associated with any cause is known as Fahr's syndrome. The causes may be hypoparathyroidism/psudo hypoparathyroidism or any other metabolic abnormality related to calcium or phosphorus metabolism. ${ }^{2}$ The other causes may be pseudopseudohypoparathyroidism, hyper/hypothyroidism,
lead/CO poisoning, tuberous sclerosis, cerebral tumour, mitochondriopathies, AIDS, TORCH infections, brucellosis, SLE, sarcoidosis and Down Syndrome. ${ }^{3}$ Fahrs disease is also known as strio-pallido-dentate calcification or calcinosis nucleorum. Basal ganglia calcification is an incidental finding in $0.3-1.2 \%$ of CT Brain scans. ${ }^{4}$

There are just a few reports of Fahr's disease in the literature. The role of FD as risk factor for FND is unknown. As far as we are aware, no case report was found in the literature which established an association between FD and FND. So, we report a sporadic case of Fahr's disease presented with functional neurological symptom disorder and discuss the possible association of this incidental neuroimaging finding with symptom presentation. 


\section{CASE PRESENTATION}

The index patient, a 17 year old girl studying in $11^{\text {th }}$ standard, belonging to a rural nuclear family of middle socioeconomic status, without any significant past or family history of psychiatric illness and medical/surgical history, presented with a duration of symptoms of 4 months which were acute in onset, characterised by episodes of abnormal in-coordinated movements of all the four limbs with her mouth deviated to right side and clenching of teeth for 5-15 minutes, preceding which she had a mild headache and altered sensation on the left side of the body. It was followed by unresponsiveness with closed eyes for 1-2 hours during which patient was always aware of her surroundings. She could hear the voices of others but was not able to respond. These episodes always occurred in front of others. History of hyperventilation was also present. She had no tongue bite, bowel and bladder incontinence, up-rolling of eye balls, no history of fall or injury during these episodes and no history of confusion was present after gaining the consciousness. Apart from these complaints, she had altered or some tingling sensations in upper extremities along with muscular cramps in legs which continued for 2-3 days and would abort without any medication. She had expressed her concerns and worries about the events only in the last one month but her distress was not persistent. Her general physical and systemic examinations were normal. Her neurological examination was also within normal limits as patient did not have any focal neurological deficit. Her mental status examination detected no abnormality except her anxious affect and the impaired attention and concentration. Her routine blood investigations like complete haemogram, random blood sugar, liver and kidney function test with electrolytes, lipid profile, thyroid profile, parathormones level, alkaline phosphatase, Vitamin D3, and viral markers (HIV, HCV, HBsAB) were found to be normal. Electroencephalogram (EEG) was normal. Computerized tomography (CT) scan of head was suggestive of bilateral symmetrical basal ganglia calcification as shown in (Figure 1). In view of the same, a magnetic resonance imaging was done, which showed nonspecific $\mathrm{T}_{2}$ flair hyperintensities in bilateral globus pallidus, periventricular white matter and prefrontal cortex. Based on clinical history and investigation results, we considered these abnormal movements as pseudoseizure or paroxysmal non-epileptic seizure (PNES). In view of the presence of sensorimotor symptoms (nonanatomical), PNES and absence of any obvious neurological symptoms, the patient was found to have a Functional Neurological Disorder (FND). The absence of these haematological or metabolic derangements rules out any secondary cause of this incidental brain

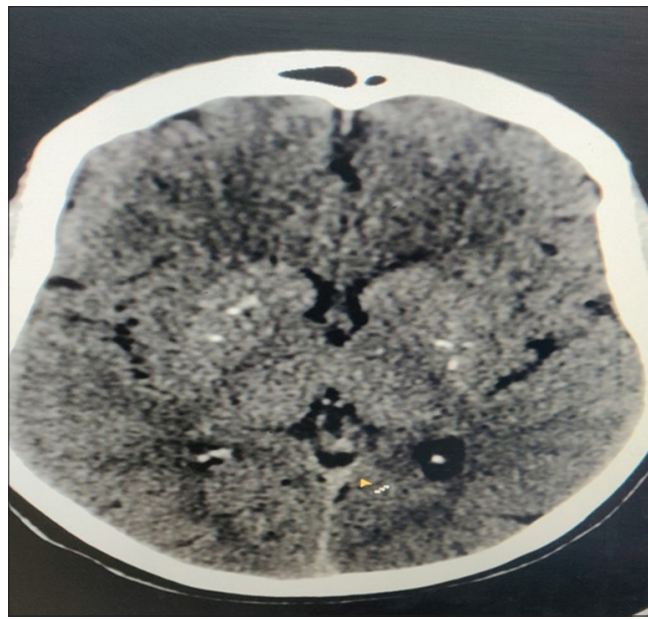

Figure 1: CT head showing bilateral basal ganglia calcification

imaging finding. Then the patient was diagnosed with Fahr's Disease.

Now, our patient was a case of functional neurological symptoms disorder along with Fahr's Disease. Then patient was managed on sertraline $50 \mathrm{mg} /$ day, clonazepam $0.75 \mathrm{mg}$ in divided doses and patient was attached with a psychologist for counselling sessions. Patient visited the outpatient clinic after the 2 weeks for follow up and reported improvement.

\section{DISCUSSION}

Based on the clinical History (waxing and waning illness with periods of remission), variable neuropsychological symptoms like non anatomical sensory loss and sleep like attacks which correspond to PNES, we made a diagnosis of FND or conversion disorder. ${ }^{5}$ Furthermore, the disorder was attributable to rare entity called Fahr's disease as no psychological factors were found in the patient.

Nearly $50-60 \%$ patients suffering from FD presents with extrapyramidal movement disorder and focal neurological deficits. ${ }^{6}$ Despite the involvement of bilateral basal ganglia, index case did not have any neurological symptoms and movement disorder. About $40 \%$ of the patients with FD present initially with psychiatric features of which mood, psychotic and cognitive symptoms occur commonly. In review of literature, both depression and mania were found to be the most common psychiatric disorders associated with FD. Some may present with anxiety disorders. ${ }^{7}$ But it was never reported about the functional neurological disorders in the cases of FD in the literature.

It is important to scrutinize the common associated biological factors in their causality where FND was not 
associated with any psychological or psychoanalytical factors and Fahr's disease can contribute to the development of FND. Patho-physiological mechanism of FND, demonstrates potential neural networks association with clinical symptoms. Basal ganglia seem to play a key role in FND. The physiological role of basal ganglia in the elaboration of movement was found to be modified during FND, with an exaggerated monitoring of limbic system interfering with motor pattern. ${ }^{8}$ In addition, other cortical structures like frontal areas and primary motor cortex may simultaneously exhibit abnormal pattern of activation, in relation to altered emotional and limbic functioning which in turn may interfere with primary motor or sensory functions (hence the symptoms productions).?

Patients with basal ganglia calcification generally have a neuropsychological dysfunction that are known to be associated with alterations of fronto-limbic-basal ganglia circuits or their inter-hemispheric connections. ${ }^{9}$ A previous study showed modification of cerebral perfusion due to hypoperfusion in basal ganglia responsible for sensory motor deficits through disruption of the cortico-basal circuits. ${ }^{10} \mathrm{~A}$ study by Hempel A et al revealed Positron Emission Tomographic (PET) findings of reduced glucose uptake, not only confined to basal ganglia, but extending to the frontal and other cortices which corresponded to the presence of neuropsychiatric deficits in a patient with Fahrs disease. ${ }^{11}$ So, the basal ganglia calcification in Fahr's disease possibly affects the similar structures which are involved in the pathophysiology of FND. This explains the common pathophysiological consortium between FND and Fahrs disease.

Index case with neuropsychiatric features and some cognitive features (impaired attention and concentration/ working memory) may suggest the widespread brain damage due to the involvement of basal ganglia and frontal lobe as MRI scan revealed the calcification in these areas. Widespread neuropsychiatric and cognitive symptoms with extensive calcification on neuroimaging has been detected by several workers in the past. ${ }^{12} \mathrm{~A}$ study by Shouyama et al also revealed that more extensive calcification, but not the distribution, correlates with the presence of higher frequency of psychiatric manifestations which could explain the presentation of the index patient with neuropsychiatric manifestations. ${ }^{13}$ Current report points to the rare association of FD, FND and extensive calcification which might be the first case reported in the literature.

\section{CONCLUSION}

In view of the rare association of FND and FD in index case, we would be vigilant in the course of disease. The diagnosis of Fahr's disease should always be taken into account in patients presenting with neuropsychiatric disturbances and meanwhile, all patients with incidentally detected basal ganglia calcifications should undergo neuropsychiatric and biochemical evaluation, so that more and more FDs may come to light with increased utilization of imaging facilities in psychiatric practice. With an appropriate use of psychotropic drugs, it is possible to manage the neuropsychiatric manifestations of FD.

\section{REFERENCES}

1. Lazar M, Ion DA, Streinu-Cercel A and Badarau AL. Fahr's syndrome: diagnosis issues in patients with unknown family history of disease. Rom J Morphol Embryol. 2009; 50(3): 425-428.

2. Dai X, Gao Y, Xu Z, Cui X, Li Y, Xu H, et al. Identification of novel genetic locus on chromosome 8p21.1-q11.23 for idiopathic basal ganglia calcification. Am J Med Genet B Neuropsychiatr Genet. 2010; 153B(7):1305-1310.

https://doi.org/10.1002/ajmg.b.31102

3. Chang R, Leung $\mathrm{CY}$ and Leong HS. Bilateral striatopallido-dentate calcinosis associated with Systemic Lupus Erythromatosus: case report and review of literature. J Neurol Sci. 2015; 358(1-2):518-519.

https://doi.org/10.1016/j.jns.2015.09.373

4. Batla A, Tai XY, Schottlaender L, Erro R, Balint B, Bhatia KP, et al. Deconstructing Fahrs disease/syndrome of brain calcification in the era of new genes. Parkinsonism Relat Disord. 2017; 37:1-10. https://doi.org/10.1016/j.parkreldis.2016.12.024

5. Carson AJ, Brown R, David AS, Duncan R, Edwards MJ, Goldstein LH, et al. Functional (conversion) neurological symptoms: Research since the millennium. J Neurol Neurosurg Psychiatry. 2012; 83(8):842-850.

https://doi.org/10.1136/jnnp-2011-301860

6. Muffaddel AA and Al-Hassani AJ. Familial idiopathic basal ganglia calcification (Fahrs disease). Netw Neurosci. 2014; 19(3):170-177.

7. Ring HA and Serra -Mestres J. Neuropsychiatry of the basal ganglia. J Neurol Neurosurg Psychiatry. 2002; 72:12-21.

https://doi.org/10.1136/jnnp.72.1.12

8. Schrag AE, Mehta AR, Bhatia KP, Brown RJ, Frackowiak RJ, Trimble MR, et al. The functional neuroimaging correlates of psychogenic versus organic dystonia. Brain. 2013; 136(3); 770-781.

https://doi.org/10.1093/brain/awt008

9. Galli S, Avbek S, Chokron S, Moulin T and Magnin E. Modifications of both functional neurological symptoms and neuroimaging patterns with good anatomoclinical concordance: a case report. BMC Neurol. 2019; 19: 270. https://doi.org/10.1186/s12883-019-1475-3

10. Boeckle M, Liegl G, Jank R and Pieh C. Neural correlates of conversion disorder: overview and meta analysis of neuroimaging studies on motor conversion disorder. BMC Psychiatry. 2016; 16:195.

https://doi.org/10.1186/s12883-016-0890-x

11. Hempel A, Henze M, Berghoff C, Garcia N, Ody R and Schroder J. PET findings and neuropsychological deficits in a case of Fahrs disease. Psychiatry Res Neuroimaging. 2001; 108:133-140. 
https://doi.org/10.1016/s0165-1781(01)00308-0

12. Srivastava S, Bhatia MS, Sharma V, Mahajan S and Rajender G. Fahr's disease: An incidental finding in a case presenting with psychosis. German J Psychiatry. 2010; 13:86-90.
13. Shouyama M, Kitabata $\mathrm{Y}$, Kaku T and Shinosaki K. Evaluation of regional cerebral blood flow in Fahr's disease with schizophrenia like psychosis: a case report. Am J Neuroradiol. 2005; 26(10):2527-2529.

\section{Author's contributions:}

AC- Concept and design of the study, Manuscript preparation, Critical revision of manuscript; SG- Literature review, Helped in preparation of first draft of manuscript and collection of image.

Work attributed to:

Bhagat Phool Singh Medical College For Women, Sonipat-131305 Haryana, India.

Orcid ID:

Dr. Sunny Garg - (D) https://orcid.org/0000-0002-5979-0241

Dr. Alka Chauhan - it https://orcid.org/0000-0001-5147-4976

Sources of support: None, Conflict of Interests: None 